\title{
In Situ Structural Analysis of Bacterial Nanomachines by Combining Cryo-FIB Milling, Cryo-ET and Sub-tomogram Analysis
}

Yunjie Chang ${ }^{1,2}$ and Jun Liu ${ }^{1,2}$

${ }^{1}$ Department of Microbial Pathogenesis, Yale University School of Medicine, West Haven, Connecticut, United States, ${ }^{2}$ Microbial Sciences Institute, Yale University, West Haven, Connecticut, United States

Cryo-electron tomography (cryo-ET) is a three-dimensional imaging technique that is suitable for imaging in situ biological structures with the potential to achieve near-atomic resolution. It has been extensively used to characterize bacterial nanomachines in situ, such as secretion systems, flagellar motors, chemoreceptors and so on. However, there are still a number of remaining challenges for in situ structural analysis of bacterial nanomachines, such as the preparation of thin samples and the symmetry mismatch between different components. In the present work, we will show our workflows for the in situ structural analysis of bacterial flagellar motor and ribosomes.

Bacterial flagellar motor is a large multi-component molecular machine, which is important for bacterial motility, mechanosensing and pathogenicity. In order to analyze the flagellar motor structure in high resolution details, we choose the causative agent of Lyme disease -- Borrelia burgdorferi (B. burgdorferi), which has a skinny cell body $(200-400 \mathrm{~nm})$ and has 7-12 motor at both cell tips. The B. burgdorferi cells were cultured to log phase, centrifuged to appropriate concentration and then plunge freeze on copper grids. The frozen-hydrated samples were transferred to a $300 \mathrm{kV}$ Titan Krios electron microscope equipped with a Direct Electron Detector (DDD) (Gatan K2 Summit) and energy filter. SerialEM[1] was used to collect the tilt series at -2 to $-4 \mu \mathrm{m}$ defocus and covering angles from $-51^{\circ}$ to $51^{\circ}$, with a tilt step of $3^{\circ}$. All recorded images were first motion-corrected using the MotionCorr2[2] and then stacked by IMOD[3]. The tilt series were aligned using fiducial markers or fiducial free alignment by IMOD. Gctf[4] was used to determine the defocus of each tilt image in the aligned stacks and the "ctfphaseflip" function in IMOD was used to do the contrast transfer function (CTF) correction for the tilt images. Tomograms were then reconstructed by weighted back-projection using IMOD[3] with the CTF corrected aligned stacks. Afterwards, the flagellar motors were manually picked from the tomograms and i3 [5, 6] was used for 3D alignment and classification. In order to analyze the stator-rotor interactions in B. burgdorferi flagellar motors, the asymmetric reconstruction of the flagellar motor was first generated with the consideration of particle distortions induced by the membrane curvature[7], then focus alignment and classification was applied to the C-ring to determine its symmetry. During the C-ring symmetry determination, the angular search range along the motor rod was restricted to be a small angle so that we can maintain overall alignment of the motor. Subsequently, all the stators in each motor were extracted to do focus alignment and classification based on membrane curvature and the C-ring density. Collectively, we were able to analyze the stator-rotor interactions in high resolution details.

Many kinds of bacteria, such as Escherichia coli (E. coli) and Salmonella enterica, are more common than spirochetes like $B$. burgdorferi but are too thick $(\sim 1 \mu \mathrm{m})$ for direct cryo-ET imaging. For these large bacteria, cryo-Focused Ion Beam (FIB) can be utilized to overcome the limitations. In present work, the wild-type $E$. coli cells were cultured to log phase, centrifuged to appropriate concentration (OD600 in range of 5-15) and then plunge freeze on copper grids. Afterwards, a cryo-FIB system (Aquilos, Thermo Fisher Scientific) was used for the milling. The plunge-frozen grids were clipped into cryo-FIB Autogrids and mounted into the specimen shuttle under liquid nitrogen. The samples were first sputter-coated with Pt $(1 \mathrm{kV}, 15 \mathrm{~mA}, 15 \mathrm{~s})$ to improve the overall sample conductivity, then deposited by an organometallic 
Pt layer (4-5 $\mu \mathrm{m}$ thick) using the gas injection system for the sample protection. Lamellae were produced using the gallium ion beam at $30 \mathrm{kV}$ with stage tilt angle around $17^{\circ}$, which results in $\mathrm{a} \sim 10^{\circ}$ angle between the final lamella and the grid. The ion beam current was reduced according to the lamella thickness $(\mathrm{t})$ during the milling process: $0.5 \mathrm{nA}$ for $\mathrm{t} \geq 3 \mu \mathrm{m}, 0.3 \mathrm{nA}$ for $\mathrm{t} \geq 1 \mu \mathrm{m}, 0.1 \mathrm{nA}$ for $\mathrm{t} \geq 700 \mathrm{~nm}, 0.05 / 0.03 \mathrm{nA}$ for final polishing. Afterwards, a thin Pt layer was sputter-coated $(1 \mathrm{kV}, 10 \mathrm{~mA}, 5 \mathrm{~s})$ on the lamella to prevent possible charging issue during cryo-ET investigation. We are able to routinely produce $\sim 100-200$ nm lamellae with such strategy. The lamellae were then transferred to Titan Krios. The tilt series collection and tomogram reconstruction were similar as that for $B$. burgdorferi flagellar motor work, but we adjust the tilt angle range based on the angle between the final lamella and the grid. Afterwards, emClarity[8] was used for 3D template search to pick the ribosomes in the tomogram and i3 [5, 6] was used for 3D alignment and classification. Ultimately, we were able to track the ribosomes and analyze the ribosome distributions in E. coli.

\section{References}

1. Mastronarde, D.N., Automated electron microscope tomography using robust prediction of specimen movements. Journal of Structural Biology, 2005. 152(1): p. 36-51.

2. Zheng, S.Q., et al., MotionCor2: anisotropic correction of beam-induced motion for improved cryoelectron microscopy. Nature Methods, 2017. 14: p. 331.

3. Kremer, J.R., D.N. Mastronarde, and J.R. McIntosh, Computer Visualization of Three-Dimensional Image Data Using IMOD. Journal of Structural Biology, 1996. 116(1): p. 71-76.

4. Zhang, K., Gctf: Real-time CTF determination and correction. J Struct Biol, 2016. 193(1): p. 1-12.

5. Winkler, H., 3D reconstruction and processing of volumetric data in cryo-electron tomography. Journal of Structural Biology, 2007. 157(1): p. 126-137.

6. Winkler, H., et al., Tomographic subvolume alignment and subvolume classification applied to myosin $V$ and SIV envelope spikes. Journal of Structural Biology, 2009. 165(2): p. 64-77.

7. Chang, Y., et al., Structural insights into flagellar stator-rotor interactions. Elife, 2019. 8. 